\title{
TREVO stent-retriever mechanical thrombectomy for acute ischemic stroke secondary to large vessel occlusion registry
}

\author{
Osama 0 Zaidat, ${ }^{1,2}$ Alicia C Castonguay, ${ }^{3}$ Raul G Nogueira, ${ }^{4}$ Diogo C Haussen, ${ }^{4}$ \\ Joey D English, ${ }^{5}$ Sudhakar R Sattii, ${ }^{6}$ Jennifer Chen ${ }^{7}$ Hamed Farid, ${ }^{8}$ Candace Borders, ${ }^{9}$ \\ Erol Veznedaroglu, ${ }_{1}^{10}$ Mandy J Binning, ${ }^{10}$ Ajit Puri, ${ }^{11}$ Nirav A Vora, ${ }_{1}^{12}$ Ron F Budzik, ${ }^{12}$ \\ Guilherme Dabus, ${ }^{13}$ Italo Linfante, ${ }^{13}$ Vallabh Janardhan, ${ }^{14}$ Amer Alshekhlee, ${ }^{15}$ \\ Michael G Abraham, ${ }^{16}$ Randall Edgell, ${ }_{1}^{17}$ Muhammad Asif Taqi, ${ }^{18}$ Ramy El Khoury, ${ }^{19}$ \\ Maxim Mokin, ${ }^{20}$ Aniel Q Majjhoo, ${ }^{21}$ Mouhammed R Kabbani, ${ }^{22}$ Michael T Froehler, ${ }^{23}$ \\ Ira Finch, ${ }^{24}$ Sameer A Ansari, ${ }^{25}$ Roberta Novakovic, ${ }^{26}$ Thanh N Nguyen ${ }^{27}$
}

For numbered affiliations see end of article.

\section{Correspondence to} Dr Osama 0 Zaidat, Director Neuroscience \& Stroke Program Mercy Health St.Vincent Hospital, Toledo 43608, Ohio, USA; ozaidat@hotmail.com

Received 17 July 2017 Revised 17 August 2017 Accepted 24 August 2017 Published Online First 29 September 2017
Check for updates

To cite: Zaidat 00 , Castonguay $A C$, Nogueira $R G$, et al. I Neurolntervent Surg 2018;10:516-524.

\section{ABSTRACT}

Background Recent randomized clinical trials (RCTs) demonstrated the efficacy of mechanical thrombectomy using stent-retrievers in patients with acute ischemic stroke (AIS) with large vessel occlusions; however, it remains unclear if these results translate to a real-world setting. The TREVO Stent-Retriever Acute Stroke (TRACK) multicenter Registry aimed to evaluate the use of the Trevo device in everyday clinical practice.

Methods Twenty-three centers enrolled consecutive AIS patients treated from March 2013 through August 2015 with the Trevo device. The primary outcome was defined as achieving a Thrombolysis in Cerebral Infarction (TICl) score of $\geq 2 b$. Secondary outcomes included 90 -day modified Rankin Scale (mRS), mortality, and symptomatic intracranial hemorrhage (sICH).

Results A total of 634patients were included. Mean age was $66.1 \pm 14.8$ years and mean baseline NIH Stroke Scale (NIHSS) score was $17.4 \pm 6.7 ; 86.7 \%$ had an anterior circulation occlusion. Mean time from symptom onset to puncture and time to revascularization were $363.1 \pm 264.5 \mathrm{~min}$ and $78.8 \pm 49.6 \mathrm{~min}$, respectively. $80.3 \%$ achieved $\mathrm{TICl} \geq 2$ b. 90 -day $\mathrm{mRS} \leq 2$ was achieved in $47.9 \%$, compared with $51.4 \%$ when restricting the analysis to the anterior circulation and within 6 hours (similar to recent AHA/ASA guidelines), and $54.3 \%$ for those who achieved complete revascularization. The 90 day mortality rate was $19.8 \%$. Independent predictors of clinical outcome included age, baseline NIHSS, use of balloon guide catheter, revascularization, and sICH.

Conclusion The TRACK Registry results demonstrate the generalizability of the recent thrombectomy RCTs in real-world clinical practice. No differences in clinical and angiographic outcomes were shown between patients treated within the AHA/ASA guidelines and those treated outside the recommendations.

\section{INTRODUCTION}

Following the publication of multiple positive consecutive randomized clinical trials (RCTs), mechanical thrombectomy is now considered the standard of care for acute ischemic stroke (AIS) due to large vessel occlusion (LVO). ${ }^{1-11}$ Improved patient selection criteria and significant technical advances leading to a higher rate of meaningful revascularization were the main advances that led to a better clinical outcome than the first-generation thrombectomy devices. ${ }^{12} 13$

However, RCTs are limited by the restrictive nature of study design in many aspects, such as inclusion and exclusion criteria, center selection, and operator experience; therefore, the results may not be generalizable to real-world practice. Post-marketing clinical registries allow for more inclusive criteria by including a range of clinical sites, operators, and varying patient populations, which provides valuable information on the generalizability and reproducibility of RCTs and additional opportunities to explore clinical hypotheses for patients treated outside these RCTs. ${ }^{14-16}$

The first stent-retriever device to be introduced to the USA was the Solitaire device, which has been shown to be as effective in achieving similar revascularization and clinical outcome in real-life post-marketing experience. Relatively comparable safety and efficacy outcomes were demonstrated in the North American Solitaire Acute ischemic stroke study (NASA Registry) and other post-marketing clinical studies. ${ }^{14-16}$ However, there is no published large-scale post-marketing investigator-initiated registry for the second FDA-approved stent-retriever, the Trevo device.

The TREVO Stent-Retriever Acute Stroke (TRACK) Registry is, to date, the largest investigator-initiated independent US post-marketing registry evaluating the real-life clinical experience of the Trevo device in 634 patients with AIS. In addition to comparing the results with the TREVO2 RCT to assess for generalizability, we also evaluated the safety and efficacy in those treated outside the AHA/ASA-published endovascular treatment guideline. $^{2}$

\section{METHODS}

Twenty-three US centers submitted retrospective and prospective data on consecutive patients with 
AIS treated from March 2013 through August 2015 with the Trevo device as the first mechanical thrombectomy treatment method to restore blood flow for LVO. Patients aged $<18$ years and those who were not treated with Trevo as the firstpass device were excluded. Sites submitted de-identified patient level data (demographic, clinical, procedural, and angiographic). Institutional review board approval was obtained at each site. Mercy Health St Vincent Hospital (Toledo, Ohio, USA) served as the coordinating center for TRACK.

\section{TRACK angiographic and clinical outcomes}

The primary angiographic outcome was defined as achieving a modified Thrombolysis in Cerebral Infarction (mTICI) score of $\geq 2 b$ at the end of the procedure. ${ }^{9}{ }^{14}$ Secondary outcomes included revascularization rate (complete revascularization to TICI 3), symptomatic intracranial hemorrhage (sICH) on the 24 hours follow-up head CT, modified Rankin Scale (mRS) score and mortality at 90 days. sICH was defined as any parenchymal hematoma, subarachnoid hemorrhage, or intraventricular hemorrhage associated with worsening of the National Institutes of Health Stroke Scale (NIHSS) score by $\geq 4$ points within 24 hours. Primary and secondary outcomes were then compared with the TREVO2 trial to assess for reproducibility of the results in the TRACK Registry.

The full TRACK cohort was further dichotomized into patients who met the AHA/ASA recommendations guidelines ${ }^{2}$ (treated $\leq 6$ hours of symptoms onset, intravenous tissue plasminogen activator (IV-tPA) (if eligible), NIHSS $\geq 6$, anterior circulation occlusion only (except for ASPECT score, which was not collected in the TRACK)) and the non-AHA/ASA population.

\section{Statistical analysis}

Statistical analyses were performed using JMP V.13 (SAS institute, Cary, North Carolina, USA). Fisher exact or $\chi^{2}$ tests were used for categorical variables and the Student's t-test $/ F$ test for continuous variables.

Baseline demographic, angiographic, and clinical outcome data were analyzed for the full TRACK cohort and compared with TREVO2 data. Key system of care and time metrics were presented for the full TRACK cohort. True First Pass Effect (tFPE), defined as TICI 3 on the first pass with no use of rescue therapy, was also evaluated.

Univariate analysis was also performed between the AHA/ASA and non-AHA/ASA groups with the TRACK cohort to compare baseline features and angiographic and clinical outcomes. For all analyses, variables with a $\mathrm{p}$ value $\leq 0.05$ and clinically relevant factors were entered into a multivariate stepwise logistic regression model to determine predictors of clinical outcome and/or mortality.

\section{RESULTS}

\section{Full TRACK cohort results}

A total of 634 consecutive patients were enrolled into the TRACK Registry (figure 1). Five patients were excluded from the final analysis $(2$ were $<18$ years of age and 3 were not treated with Trevo as the first device).

\section{Demographic and baseline features}

Baseline demographics are shown in table 1. Mean baseline NIHSS was higher in TREVO2 $(18.3 \pm 5.3)$ than in TRACK

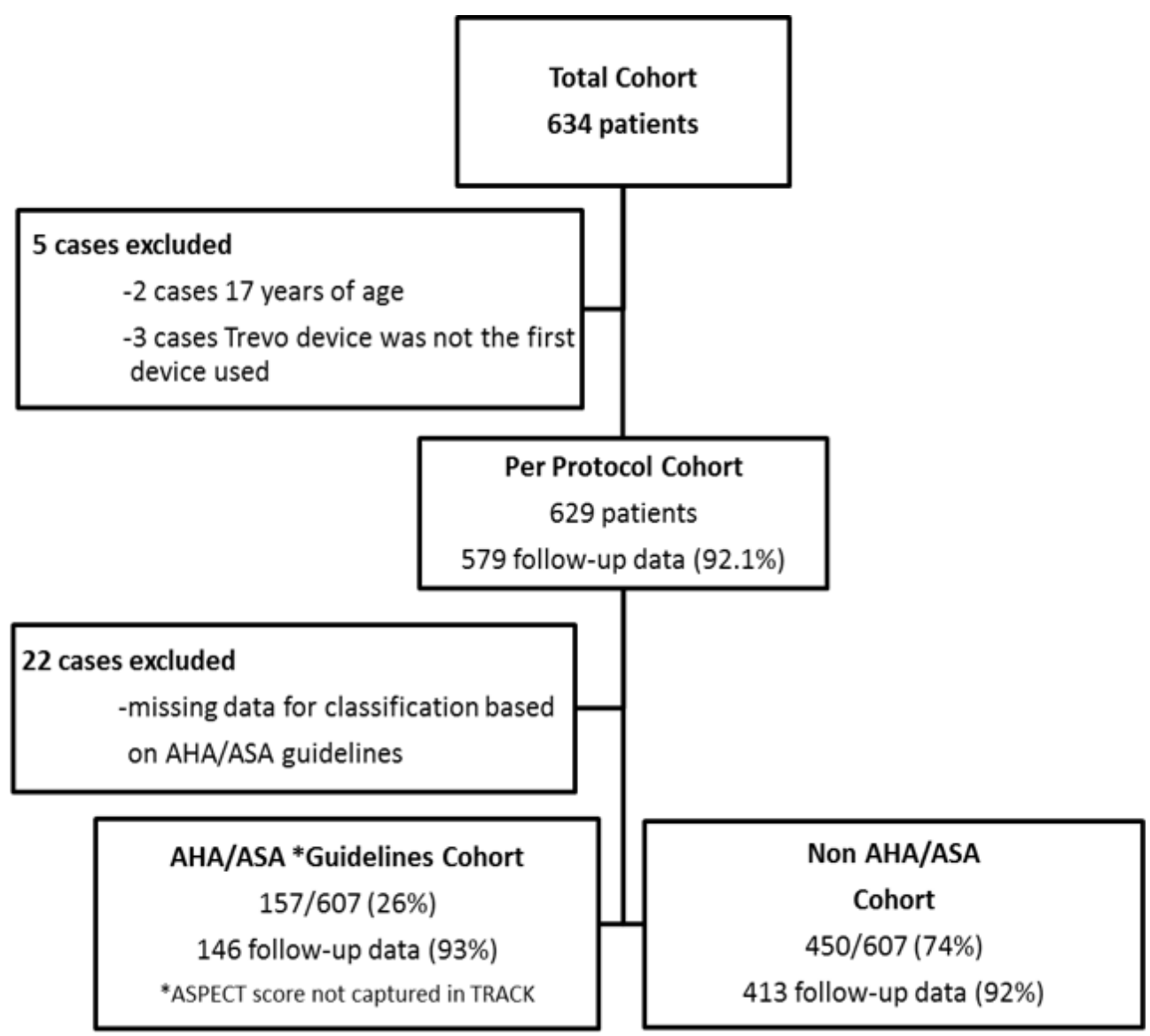

Figure 1 TRACK Registry Flow Chart. 
Table 1 Baseline and procedural characteristics of the TRACK Registry compared with TREV02 and TRACK AHA/ASA and non-AHA/ASA subcohorts

\begin{tabular}{|c|c|c|c|c|c|c|}
\hline & $\begin{array}{l}\text { TRACK } \\
(\mathrm{n}=629) \\
\mathrm{N}(\%)\end{array}$ & $\begin{array}{l}\text { TREVO2 } \\
(\mathrm{n}=88) \\
\mathrm{N}(\%)\end{array}$ & $\begin{array}{l}\mathrm{p} \text { Value } \\
\text { TRACK versus } \\
\text { TREVO2 }\end{array}$ & $\begin{array}{l}\text { TRACK-AHA/ASA } \\
(n=157) \\
N(\%)\end{array}$ & $\begin{array}{l}\text { TRACK-non-AHA/ASA } \\
(n=450) \\
N(\%)\end{array}$ & $\begin{array}{l}p \text { Value } \\
\text { AHA/ASA versus } \\
\text { non-AHA/ASA }\end{array}$ \\
\hline \multicolumn{7}{|l|}{ Demographics } \\
\hline Age (years), mean (SD) & $66.1(14.8)$ & $67.4(13.9)$ & 0.24 & $64.9(15.5)$ & $66.5(14.5)$ & 0.24 \\
\hline Age $>80$ & $114(18.1)$ & NA & NA & $27(17.2)$ & 85 (18.9) & 0.72 \\
\hline Gender (female) & $305(48.3)$ & $48(55)$ & 0.3 & $71(45.2)$ & $220(48.9)$ & 0.46 \\
\hline Race (white) & $424(68.1)$ & NA & NA & $101(64.3)$ & $305(69.0)$ & 0.09 \\
\hline \multicolumn{7}{|l|}{ Vascular risk factors } \\
\hline Hypertension & $473(75.0)$ & $67(76)$ & 0.04 & $124(79.0)$ & $335(74.4)$ & 0.28 \\
\hline Atrial fibrillation & 247 (39.2) & $42(48)$ & 0.13 & $56(35.7)$ & $184(41.0)$ & 0.26 \\
\hline Diabetes mellitus & $161(25.5)$ & $33(38)$ & 0.02 & $41(26.1)$ & $117(26.0)$ & 1.00 \\
\hline Hyperlipidemia & $314(49.8)$ & $55(63)$ & 0.03 & $76(48.4)$ & $229(50.2)$ & 0.64 \\
\hline Smoking history & $154(24.5)$ & $37(42)$ & 0.001 & $40(25.5)$ & $112(25.0)$ & 0.92 \\
\hline Coronary artery disease & $146(29.4)$ & $29(33)$ & 0.1 & $35(28.2)$ & $104(29.6)$ & 0.82 \\
\hline \multicolumn{7}{|l|}{ Clinical presentation } \\
\hline Baseline mRS (median) (min, max) & $0(0,5)$ & $0(0,1)$ & & $0(0,1)$ & $0(0,5)$ & \\
\hline Initial NIHSS, mean (SD) & $17.4(6.7)$ & $18.3(5.3)$ & 0.04 & $18.0(5.8)$ & $17.2(6.9)$ & 0.19 \\
\hline \multicolumn{7}{|l|}{ Occlusion site } \\
\hline All MCA segments & $434(68.9)$ & $67(76)$ & 0.002 & $118(75.2)$ & $298(66.4)$ & 0.06 \\
\hline M1 & $344(54.6)$ & $53(60)$ & 0.03 & $118(75.2)$ & $209(70.1)$ & 0.0001 \\
\hline M2 & $84(13.3)$ & $14(16)$ & 0.5 & $0(0)$ & $83(27.9)$ & 0.0001 \\
\hline M3 & $6(1.0)$ & $0(0)$ & 0.2 & $0(0)$ & $6(2.0)$ & 0.34 \\
\hline ICA & $100(15.9)$ & $14(16)$ & 1.0 & $39(24.8)$ & $55(12.3)$ & 0.0003 \\
\hline Vertebrobasilar circulation & $84(12.7)$ & $7(8)$ & 0.2 & $0(0)$ & $84(18.7)$ & 0.0001 \\
\hline Anterior circulation & $546(86.7)$ & $81(92)$ & 0.2 & $157(100)$ & $365(81.3)$ & 0.0001 \\
\hline Initial systolic BP, mean (SD) & $144.9(26.6)$ & NA & NA & $145.9(28.1)$ & $144.7(26.2)$ & 0.68 \\
\hline Initial diastolic BP, mean (SD) & $78.2(19.2)$ & NA & NA & $78.6(20.9)$ & $78.1(18.6)$ & 0.63 \\
\hline IV tPA & $321(51.2)$ & $51(58)$ & 0.22 & $157(100)$ & $164(34.9)$ & 0.0001 \\
\hline Hospital transfer & $315(50.0)$ & NA & NA & $62(39.5)$ & $237(52.8)$ & 0.005 \\
\hline \multicolumn{7}{|l|}{ Procedural factors } \\
\hline Time from onset to puncture (min), mean (SD) & $363.1(264.5)$ & $276(90)$ & 0.00 & $217.5(67.8)$ & $413.8(287.7)$ & $<0.0001$ \\
\hline General anesthesia & $394(62.4)$ & $72(82)$ & 0.004 & $89(56.7)$ & $284(63.1)$ & 0.2 \\
\hline Regional aspiration & $142(22.6)$ & NA & NA & $44(30.3)$ & $98(23.6)$ & 0.12 \\
\hline IA tPA & $130(20.7)$ & NA & NA & $46(29.3)$ & $83(18.5)$ & 0.006 \\
\hline Balloon guide catheter & $298(47.3)$ & NA & NA & $84(53.5)$ & $206(45.9)$ & 0.08 \\
\hline
\end{tabular}

Bold type indicates statistical significance.

AHA, American Heart Association; ASA, American Stroke Association; ICA, internal carotid artery; mRS, modified Rankin Scale; MCA, middle cerebral artery; NIHSS, National Institutes of Health Stroke Scale; tPA, tissue plasminogen activator.

$(17.4 \pm 6.7)(\mathrm{p}=0.04)$. TRACK had a significantly lower proportion of patients with diabetes, hyperlipidemia, and smoking history. The majority of patients presented with an anterior circulation occlusion (86.7\%), with less middle cerebral artery (MCA) occlusion in TRACK compared with TREVO2 (68.9\% vs $76 \%, \mathrm{p}=0.0002$ ).

TRACK time metrics

Key time metrics in TRACK are presented in figure $2 \mathrm{~A}$ and show a median last known well to presentation at the treating hospital door of $160 \mathrm{~min}$, a door to puncture time of $118 \mathrm{~min}$, and final puncture to successful recanalization to TICI $\geq 2 \mathrm{~b}$ of $67 \mathrm{~min}$.
The majority of TRACK patients $(48 \%)$ had their puncture within 4.5 hours from onset while $15 \%$ had their puncture at 4.5-6 hours (figure 2B).

Primary angiographic outcomes

The TRACK operator-adjudicated revascularization rates of TICI $\geq 2 \mathrm{a}$, TICI $\geq 2 \mathrm{~b}$, and TICI 3 were significantly higher than in TREVO2 $(92.8 \%, 80.3 \%, 44.5 \%$ and $85 \%, 68 \%, 14 \%$ in TRACK and TREVO2, respectively) (table 2 ). The TICI $\geq 2 \mathrm{~b}$ revascularization rate post-Trevo device use only was $68.8 \%$ in TRACK versus 68\% in TREVO2. 
A
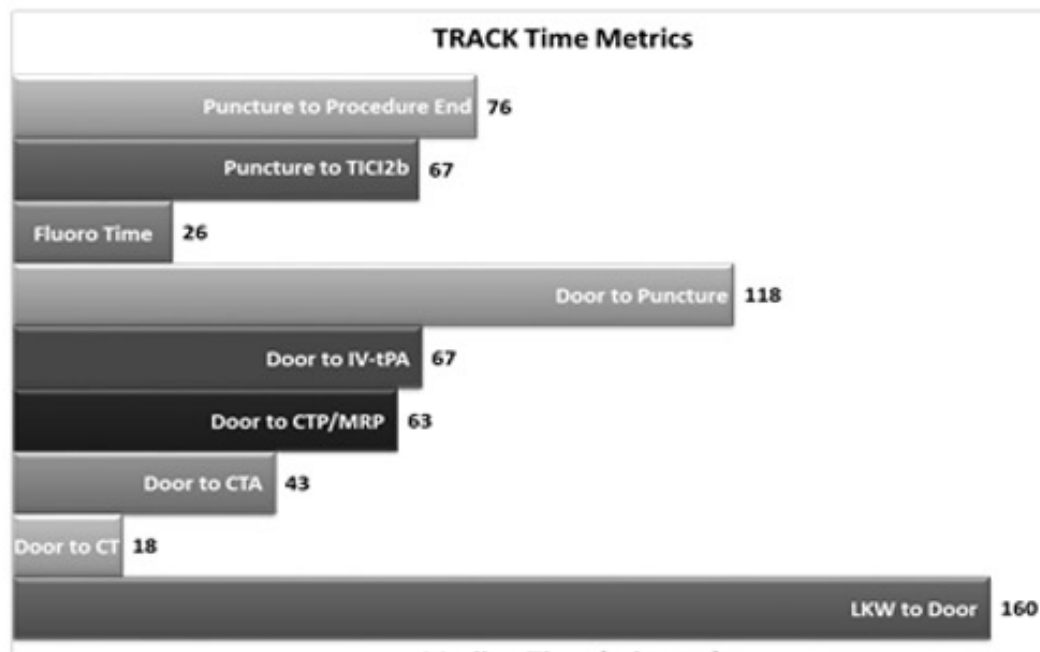

Median Time (minutes)

B

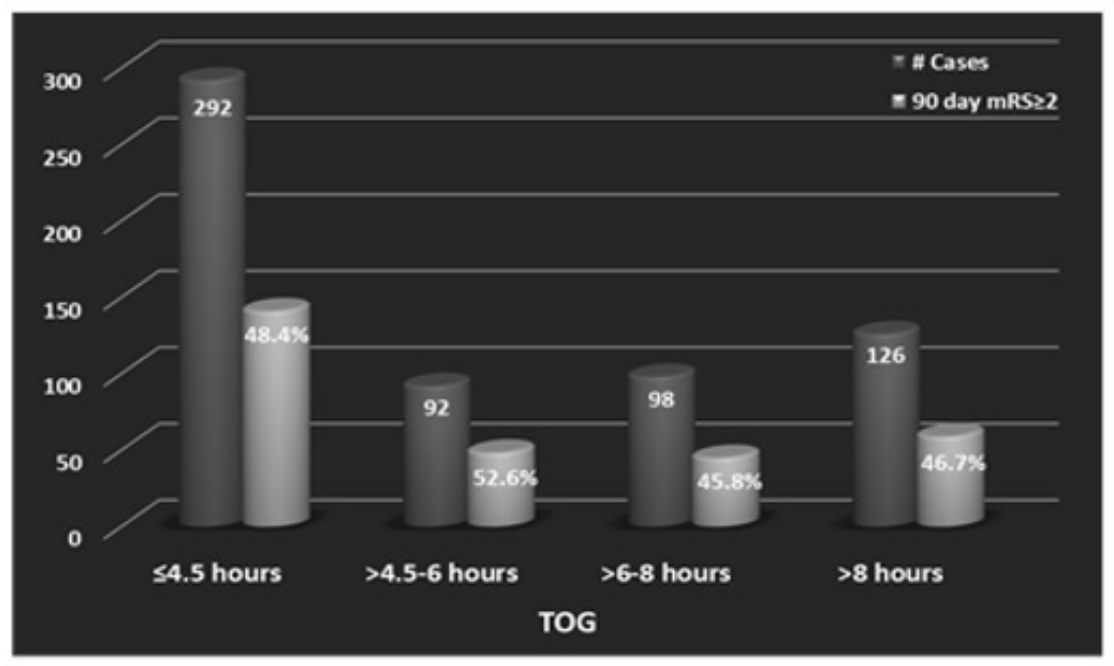

C

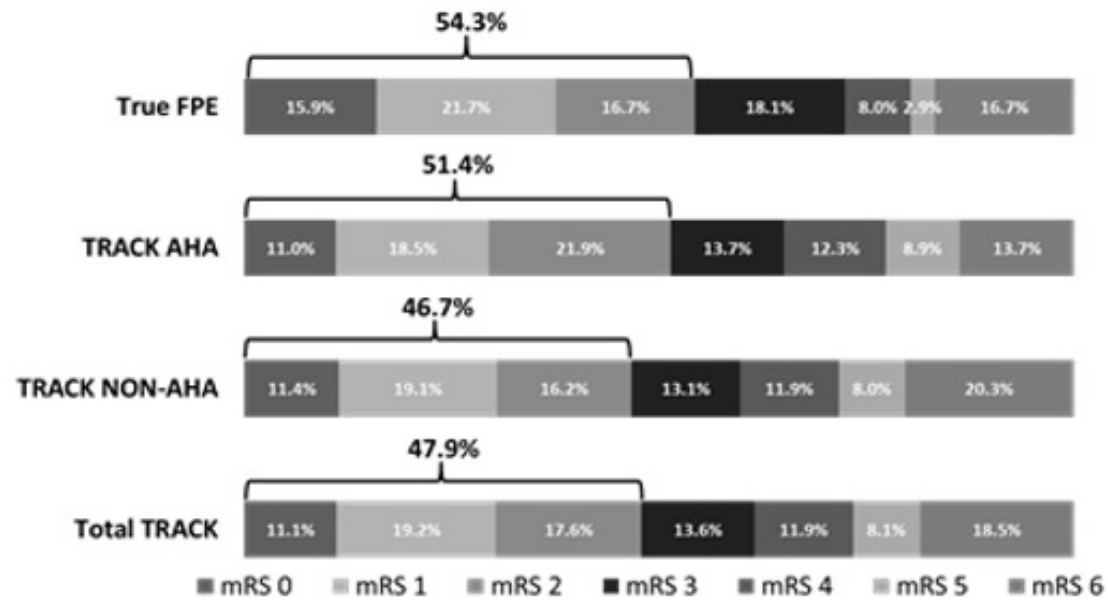

Figure 2 TRACK Registry key time metrics and outcomes. (A) Key time metrics. (B) Distribution of patients according to Tme of Onset to Groin puncture (TOG) and percentage of good clinical outcome in each stratum. (C) Distribution of 90-day modified Rankin Scale (mRS) scores in the TRACK Registry, AHA/ASA, non-AHA/ASA, and true First Pass Effect (tFPE) cohorts. The bar indicates patients with a good clinical outcome (mRS $\leq 2$ ). tFPE is defined as $\mathrm{TICl} 3$ on the first pass with no use of rescue therapy.

The mean number of passes in TRACK was $1.9 \pm 1.2$ compared with $2.4 \pm 1.4$ in TREVO2 $(\mathrm{p}=0.002)$. The majority of TRACK cases $(45.6 \%)$ were treated using a single device pass.
Rescue therapy was used in $21.5 \%$ of TRACK patients. Operator-adjudicated rates of distal embolization and embolization into new territory in TRACK were $23 \%$ and $4.5 \%$, respectively. 


\begin{tabular}{|c|c|c|c|c|c|c|}
\hline & $\begin{array}{l}\text { TRACK }(n=629) \\
\text { N }(\%)\end{array}$ & $\begin{array}{l}\text { TREVO2 } \\
(\mathrm{n}=88) \mathrm{N}(\%)\end{array}$ & $\begin{array}{l}p \text { Value TRACK } \\
\text { versus TREV02 }\end{array}$ & $\begin{array}{l}{ }^{*} \text { TRACK-AHA/ASA } \\
(\mathrm{n}=157) \mathrm{N}(\%)\end{array}$ & $\begin{array}{l}\text { *TRACK-non-AHA/ASA } \\
(\mathrm{n}=450) \\
\mathrm{N}(\%)\end{array}$ & $\begin{array}{l}{ }^{*} p \text { Value } \\
\text { AHA/ASA } \\
\text { versus non- } \\
\text { AHA/ASA }\end{array}$ \\
\hline \multicolumn{7}{|l|}{ Clinical outcome } \\
\hline $\mathrm{mRS} \leq 2$ at 90 days & $277(47.9)$ & $34(40)$ & 0.2 & $75(51.4)$ & $\begin{array}{l}193(46.7) \\
\text { Anterior circulation only: } \\
158 / 333(47.5 \%)\end{array}$ & $\begin{array}{l}0.34 \\
0.49\end{array}$ \\
\hline NIHSS at discharge, mean (SD) & $17.4(6.7)$ & NA & NA & $11.3(11.3)$ & $11.3(11.1)$ & 1.00 \\
\hline NIHSS at 90 days, mean (SD) & $18.1(18.7)$ & NA & NA & $13.7(16.6)$ & $\begin{array}{l}19.9(19.3) \\
\text { Anterior circulation } \\
\text { only: } 18.9(19)\end{array}$ & $\begin{array}{l}0.04 \\
0.1\end{array}$ \\
\hline Mortality at 90 days & $106(19.8)$ & $29(33)$ & 0.01 & $19(14.1)$ & $\begin{array}{l}85(22.2) \\
\text { Anterior circulation only: } \\
59(19.3)\end{array}$ & $\begin{array}{l}0.05 \\
0.22\end{array}$ \\
\hline $\mathrm{sICH}$ & $44(7.1)$ & $4(4.5)$ & 0.4 & $8(5.2)$ & $35(8.0)$ & 0.28 \\
\hline \multicolumn{7}{|l|}{ Angiographic outcomes } \\
\hline $\mathrm{TIMI} \geq 2$ & $564(89.7)$ & NA & NA & $141(89.8)$ & $400(89.6)$ & 1.0 \\
\hline $\mathrm{TICl} \geq 2 \mathrm{a}$ & $584(92.8)$ & $73(85)$ & 0.0004 & $146(93.0)$ & $415(92.9)$ & 1.0 \\
\hline $\mathrm{TICl} \geq 2 \mathrm{~b}$ & $505(80.3)$ & $60(68)^{*}$ & 0.005 & $128(81.5)$ & $360(80.4)$ & 0.81 \\
\hline $\mathrm{TICl} 2 \mathrm{a}$ & $79(12.6)$ & $19(22)^{*}$ & 0.004 & $19(12.1)$ & $60(12.7)$ & 0.89 \\
\hline $\mathrm{TICl} 2 \mathrm{~b}$ & $225(35.8)$ & $48(54)^{*}$ & 0.001 & $45(28.7)$ & $180(38.1)$ & 0.03 \\
\hline $\mathrm{TICl} 3$ & $280(44.5)$ & $12(14)^{*}$ & $<0.05$ & $82(52.2)$ & $198(41.9)$ & 0.03 \\
\hline Distal embolization & $112(23.0)$ & NA & NA & $24(19.4)$ & $81(23.7)$ & 0.38 \\
\hline Embolization into new territory & $20(4.5)$ & NA & NA & $10(8.3)$ & $9(2.9)$ & 0.042 \\
\hline No of passes, mean (SD) & $1.9(1.2)$ & $2.4(1.4)$ & 0.002 & $2.0(1.1)$ & $1.9(1.2)$ & 0.40 \\
\hline 1 & $272(45.6)$ & NA & NA & $61(41.2)$ & $201(47.1)$ & 0.22 \\
\hline 2 & $167(28.2)$ & NA & NA & $44(29.7)$ & $123(27.5)$ & 0.92 \\
\hline 3 & $112(18.8)$ & NA & NA & $34(23.0)$ & $78(17.4)$ & 0.23 \\
\hline$>3$ & $45(7.5)$ & NA & NA & $6(4.1)$ & $36(8.0)$ & 0.10 \\
\hline Use of rescue therapy & $133(21.5)$ & $16(18)$ & 0.48 & $35(22.9)$ & $93(21.0)$ & 0.65 \\
\hline \multicolumn{7}{|l|}{ Type of rescue therapy $(65 / 133)$} \\
\hline Solitaire & $29(44.6)$ & & & & & \\
\hline IA-tPA & $28(43.1)$ & & & & & \\
\hline Aspiration & $41(63.1)$ & & & & & \\
\hline Intracranial angioplasty & $15(23.1)$ & & & & & \\
\hline Fluoroscopic time (min) & $32.3(22.9)$ & NA & NA & $31.9(21.2)$ & $32.8(23.8)$ & 0.35 \\
\hline $\begin{array}{l}\text { Time of puncture to revascularization or end of } \\
\text { procedure (min), mean (SD) }\end{array}$ & $78.8(49.6)$ & NA & NA & $77.1(46.8)$ & $79.2(48.9)$ & 0.64 \\
\hline
\end{tabular}

${ }^{*}$ Core laboratory adjudicated.

Bold type indicates statistical significance.

AHA, American Heart Association; ASA, American Stroke Association, mRS, modified Rankin Scale; NIHSS, National Institutes of Health Stroke Scale; sICH, symptomatic intracranial hemorrhage; $\mathrm{TICl}$, Thrombolysis In Cerebral Infarction; tPA, tissue plasminogen activator.

\section{Primary clinical outcomes}

Ninety-day clinical outcome data were available in $92 \%$ (579/629). Good clinical outcome (defined as $\mathrm{mRS} \leq 2$ at 90 days) was achieved in $48 \%$ of TRACK patients (table 2 and figure $2 \mathrm{C}$ ). No difference in sICH rate was observed between TRACK and TREVO2. The mortality rate was lower in TRACK than in TREVO2 $(19.8 \%$ vs $33 \%, \mathrm{p}=0.01)$.

Finally, we assessed the rate of good clinical outcome in those who achieved tFPE of TICI 3 without the use of adjunctive therapy (25\%). This group achieved a higher rate of good clinical outcome $(54.3 \%)$ than the rate of $48 \%$ achieved for the overall TRACK cohort $(\mathrm{p}<0.05)$ (figure $2 \mathrm{C}$ ).
Predictors of clinical outcomes

On univariate analysis, the main predictors of good outcome (mRS 0-2) were recanalization success, use of balloon guide catheter (BGC), and tFPE, and predictors of poor outcome (3-6 mRS) included age, NIHSS, hypertension, hyperlipidemia, diabetes, procedure time, and sICH (figure 3). However, after multivariate adjustment, the variables that remained as independent predictors of clinical outcome were age, initial NIHSS, BGC, sICH, and tFPE.

Additionally, we performed unadjusted analyses of key clinical and technical factors known to influence mRS. Figure 4A-D demonstrates an inverse correlation between clinical outcome 


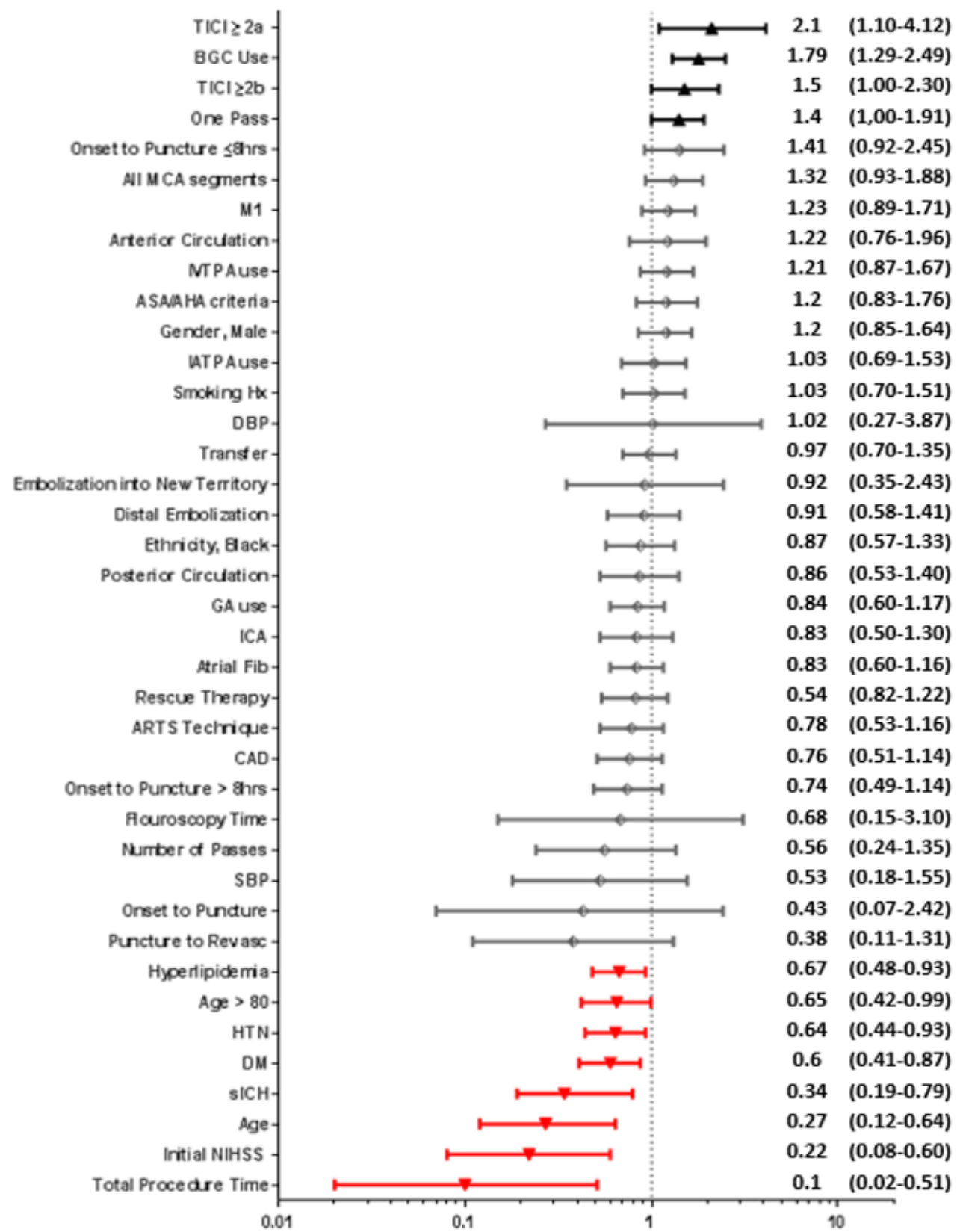

Figure 3 Forest plot of univariate predictors of clinical outcome dichotomized as mRS $0-2.0 R(95 \% \mathrm{Cl})$ are depicted to the right of the figure. Red and black bars indicate variables which are significant.

and age, baseline NIHSS, time to revascularization, and number of passes. The highest probability of achieving $\mathrm{mRS} 0-2$ is patients of younger age with lower baseline NIHSS, shorter time to revascularization, and fewer number of passes, while those with advanced age, higher initial NIHSS, longer time to revascularization, and high number of passes had the greatest probability of achieving an mRS 4-6. These factors did not appear to influence the probability of achieving mRS 3 .

\section{TRACK AHA and non-AHA subgroups}

For the TRACK AHA and non-AHA cohort analysis, 22 cases were excluded due to missing data necessary for classification based on AHA/ASA guidelines; 157 and 450 patients met the criteria for the AHA/ASA and non-AHA/ASA groups, respectively (figure 1).
Baseline variables

Given the exclusivity of anterior circulation lesions in the AHA/ ASA cohort, there was a statistical difference between the AHA/ASA and non-AHA/ASA groups in the occlusion location (table 1). Mean time from onset to puncture was different between the AHA/ASA and non-AHA/ASA groups $(217.5 \pm 67.8 \mathrm{~min}$ vs $413.8 \pm 287.7 \mathrm{~min}$, $\mathrm{p}<0.0001)$. IV-tPA use was $100 \%$ versus $34.9 \%$ in the AHA/ASA and non-AHA/ASA cohorts, respectively. Patient transfer was more common in the non-AHA/ASA group $(53 \%, \mathrm{p}=0.005)$. A higher rate of IA thrombolytic utilization was noted in the AHA/ASA group $(29 \%, \mathrm{p}=0.006)$.

Angiographic and technical outcomes

No difference was observed in revascularization success rates in the AHA/ASA and non-AHA/ASA groups (table 2). However, a significantly higher rate of TICI 3 was seen in the AHA/ASA 

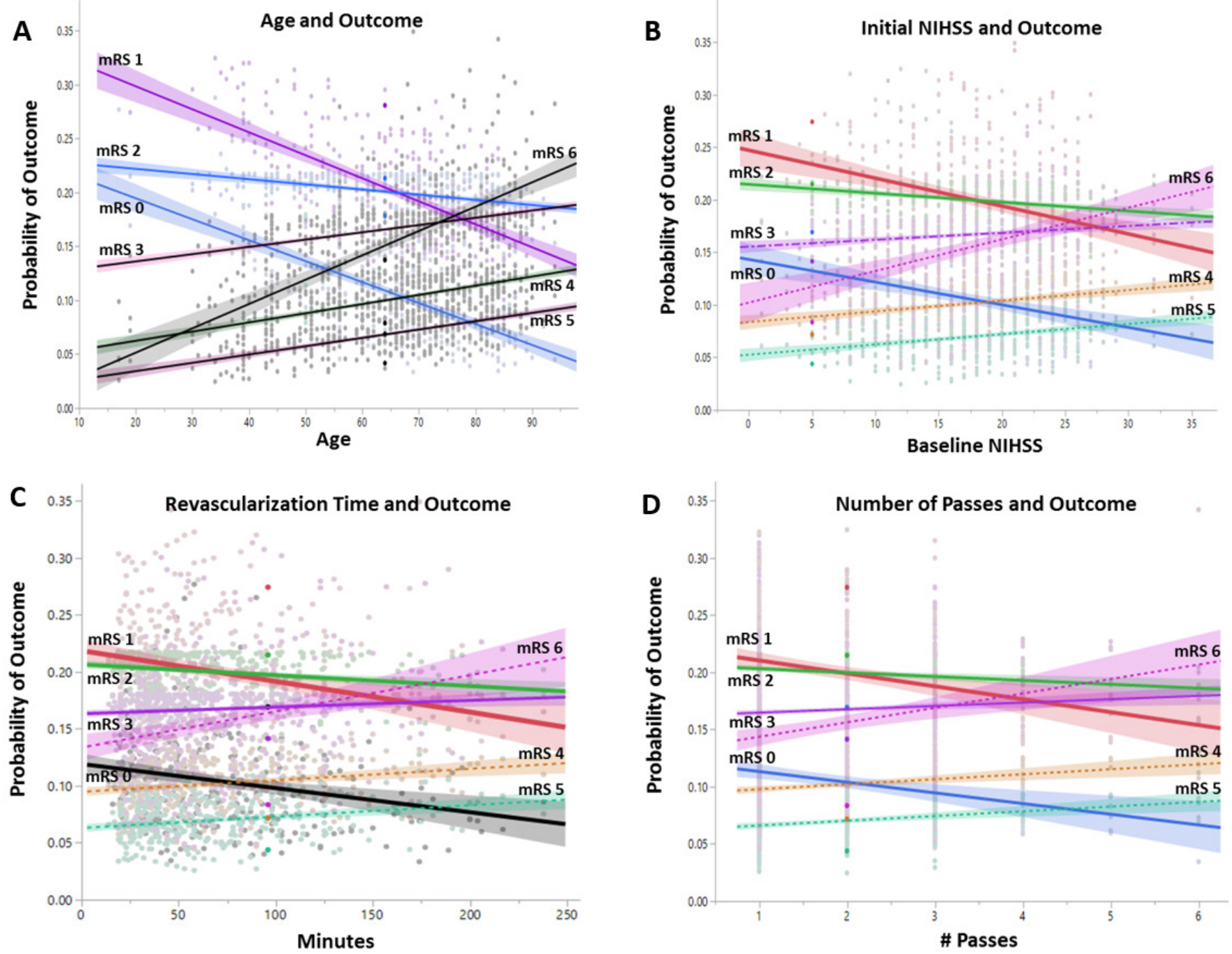

Figure 4 (A-D) Probability of ordinal modified Rankin Scale (mRS) with key clinical and technical factors. Note the highest probability of mRS 1 with younger age, lower initial NIHSS, shorter revascularization time, and smaller number of passes; mRS 3 is not a discriminator. An inverse effect was noted at the other extremes, with a higher probability of mRS 6 with older age, higher initial NIHSS, longer revascularization time, and higher number of passes.

cohort. Embolization into new territory was higher in AHA/ASA patients $(8.3 \%$ vs $2.9 \%, \mathrm{p}=0.04)$.

Clinical outcomes

The rate of good clinical outcome at 90 days (mRS $\leq 2$ ) was similar between the cohorts but mortality was higher in the non-AHA/ASA group (table 2).

\section{DISCUSSION}

To date, TRACK is the largest investigator-initiated, real-life, post-marketing, clinical registry evaluating a total of 634 subjects treated with Trevo as the first device for AIS secondary to LVO. Results from the TRACK Registry supported similar angiographic and clinical outcomes as reported in TREVO2 RCTs and the NASA Registry. ${ }^{9} 10$

A unique observation from this real-life registry is that a large proportion of patients $(74.1 \%)$ were treated outside the recent AHA/ASA guidelines and recommendations for endovascular therapy. For example, $18.1 \%$ in the non-AHA/ASA group were $>80$ years of age and $37 \%$ of patients were outside the
6 hour time window; however, these subgroups still had an excellent angiographic and clinical outcome (table 2, figure 2 b).

\section{Angiographic outcomes}

The final angiographic revascularization outcome (after all devices and rescue therapy) of TICI $\geq 2 b$ was higher in TRACK than in TREVO2 $(80.3 \%$ vs $68 \%) .{ }^{9}$ However, when comparing the revascularization rate post-Trevo device use only, there was no difference between the two cohorts $(68.8 \%$ in TRACK versus $68 \%$ in TREVO2). ${ }^{9}$ When compared with the recently published randomized trials, the rate of TICI $2 \mathrm{~b} / 3$ is comparable with the Solitaire-treated patients in the SEER pooled analysis (77\%) and with the HERMES mechanical thrombectomy group (71\%). ${ }^{11}$

In our study, we observed a significantly higher proportion of patients achieving near complete final revascularization to TICI 3 (44.5\% final and $37.4 \%$ post-Trevo only vs $14 \%$ in TREVO2). ${ }^{9}$ The higher rate of near complete revascularization may be explained by several factors. (1) In real-life experience there is no limit to the number of passes that an operator can attempt. (2) The rate of BGC use, proven since the TREVO2 
study to be associated with a higher rate of near complete revascularization, may be higher in the current registry $(47.3 \%$ in TRACK vs $44.1 \%$ in NASA, unavailable in TREVO2 study). ${ }^{15} 17$ (3) An improvement in operator learning and experience since the approval of the device. (4) The combined approach of Trevo device with local aspiration from a distal large-bore catheter, which was used in approximately $23 \%$ of the cases.

Although potential for self-reporting bias of the angiographic data is another valid reason for the higher rate of near complete revascularization, TREVO2 showed that there was no difference between operator and core laboratory adjudication of the recanalization. ${ }^{9}$

The self-reported rates of distal embolization and embolization into new territory were similar to those in the NASA Registry (23\% vs $16.4 \%$ and $4.5 \%$ vs $5.5 \%$, respectively). ${ }^{10}$ The rate of distal embolization correlated well with those who did not achieve TICI $2 \mathrm{~b}$ or higher, indicating possible persistent downstream occlusion. The ability to accurately estimate the rate of new embolization is limited by lack of complete baseline angiographic assessment or availability of pre-intervention CT angiographic data.

\section{Clinical outcome}

Despite the high rate of posterior circulation thrombectomy (table 1) and percentage of patients who had an onset to puncture time $>6$ hours (figure $2 \mathrm{~b}$ ), the rate of good clinical outcome in the TRACK Registry was $47.9 \%$, which compared well with the rates in TREVO2 (40\%) and with the NASA Registry (42\%). ${ }^{910}$

In the TRACK AHA/ASA group the rate of a good clinical outcome was $51.4 \%$, which is similar to the rates of good clinical outcome noted in the HERMES and SEER pooled analyses (46\% and $54 \%$, respectively). ${ }^{11} 12$ The clinical outcome in TRACK was also augmented to $54.3 \%$ in patients achieving tFPE to TICI 3 (figure 3), as shown in previous studies. ${ }^{17}$

The rates of sICH were similar in TRACK (7.1\%), TREVO2 (4.1\%), and NASA (9.9\%)..$^{9} 10$ The numerical difference in hemorrhage rate in TRACK versus TREVO2 may be related to a more liberal inclusion of patients with severe strokes and those outside the 8 hour time window. When patients with only anterior circulation were evaluated, the rate of symptomatic hemorrhage decreased to $5.2 \%$, which is similar to the HERMES pooled analysis of $4.4 \% .^{12}$

The mortality rate in TRACK was $19.8 \%$, which is lower than the reported rate of $33 \%$ in NASA. ${ }^{9}{ }^{10}$ When examining the mortality rate within the AHA/ASA guidelines-like group, the mortality rate decreased to $14.4 \%$, which compared well with the $15.3 \%$ noted with the HERMES pooled analysis. ${ }^{12}$

Similar to other studies, recanalization, FPE, BGC, and MCA clot location predicted a favorable outcome while age, procedure time, symptomatic hemorrhage, initial NIHSS and age were predictors of a poor clinical outcome as depicted. ${ }^{18-20}$

An interesting observation was noted in the ordinal mRS outcome of the key predictors of clinical outcome (figure 4). We noted a higher likelihood of achieving mRS 1 , followed by 2 then 0 with endovascular therapy, when the predictors favored a good outcome (such as shorter onset to puncture time, younger age, less severe baseline NIHSS, shorter revascularization time, and fewer number of device attempts). mRS 6 , followed by 4 , then 5 (in this order) were more likely to happen when these factors were not favorable for a good clinical outcome. Interestingly, mRS 3 was flat with no slope and predication ability across all these clinical variables.

Revascularization time (defined as puncture to TICI $2 \mathrm{~b}$ ) metrics in TRACK showed a mean time of $78 \pm 49.6 \mathrm{~min}$ compared with $77.1 \pm 46.8 \mathrm{~min}$ in NASA. ${ }^{10}$ This may be related to individual patient variability between the two studies and may be evaluated in the future in an individual patient-level pooled analysis. An additional potential explanation is the difference in centers participating in the two studies and their system of care and local processes.

\section{Limitations}

TRACK has the inherent limitation of self-reported prospective and retrospective angiographic and clinical outcome data. However, site and core laboratory-reported angiographic outcomes in TREVO2 were similar. In addition, including all consecutive patients and sample size may have reduced the selection bias.

Another limitation is the potential lack of precision in our reported time metrics due to the retrospective nature of the data, which could result in over/underestimation of time metrics (such as the door to puncture and revascularization times).

\section{CONCLUSION}

Our real-life experience with the TREVO device demonstrated the ability to mirror the recanalization, safety, and efficacy outcomes obtained in the TREVO2, SEER, and HERMES randomized clinical studies.

\section{Author affiliations}

${ }^{1}$ Departments of Endovascular Neurosurgery and Stroke, St Vincent Mercy Medical Center, Toledo, Ohio, USA

${ }^{2}$ Neuroscience \& Stroke Center, Mercy Health St Vincent Hospital, Toledo, OH, USA

${ }^{3}$ Department of Biostatistics, University of Texas, Houston, Texas, USA

${ }^{4}$ Department of Neurology, Emory University School of Medicine, Atlanta, Georgia, USA

${ }^{5}$ California Pacific Medical Center, Atlanta, Florida, USA

${ }^{6}$ Department of Neurointerventional Surgery, Christiana Care Health System, Newark, Delaware, USA

${ }^{7}$ Sidney Kimmel Medical College, Philadelphia, Pennsylvania, USA

${ }^{8}$ Department of Neurointerventional Radiology, St Jude Medical Center, Fullerton, USA

${ }^{9}$ University of California, Irvine School of Medicine, Irvine, California, USA

${ }^{10}$ Department of Neurosurgery, Drexel Neurosciences Institute, Philadelphia,

Pennsylvania, USA

${ }^{11}$ Department of Radiology, University of Massachusetts Medical School, Worcester,

Massachusetts, USA

${ }^{12}$ Department of Radiology, Riverside Radiology and Interventional Associates,

Columbus, Ohio, USA

${ }^{13}$ Department of Neurointerventional Surgery, Baptist Cardiac and Vascular Institute, Miami, Florida, USA

${ }^{14}$ Texas Stroke Institute, Plano, Texas, USA

${ }^{15}$ Department of Vascular and Interventional Neurology, DePaul Stroke Center-SSM

Neuroscience Institutes, St Louis University, St Louis, Missouri, USA

${ }^{16}$ Departments of Neurology and Interventional Radiology, University of Kansas

Medical Center, Kansas, USA

${ }^{17}$ Department of Neurology, St Louis University, St Louis, Missouri, USA

${ }^{18}$ Department of Neurology and Neurosurgery, Los Robles Hospital and Medical

Center, Thousand Oaks, California, USA

${ }^{19}$ Department of Neurology, Tulane University, New Orleans, Louisiana, USA

${ }^{20}$ Department of Neurosurgery and Brain Repair, University of South Florida, Tampa,

Florida, USA

${ }^{21}$ Department of Neurology, Wayne State School of Medicine, Detroit, Michigan, USA

${ }^{22}$ Department of Neurosurgery, Gundersen Health System, La Crosse, Wisconsin, USA

${ }^{23}$ Departments of Neurology, Neurosurgery, and Radiology, Vanderbilt University

Medical Center, Nashville, Tennessee, USA

${ }^{24}$ John Muir Health, Walnut Creek, California, USA

${ }^{25}$ Departments of Radiology, Neurology, and Neurological Surgery, Northwestern

University, Feinberg School of Medicine, Chicago, Illinois, USA

${ }^{26}$ Departments of Radiology, Neurology, and Neurotherapeutics, UT Southwestern Medical Center, Dallas, Texas, USA

${ }^{27}$ Department of Neurology, Neurosurgery, and Radiology, Boston Medical Center, Boston, Massachusetts, USA

Contributors All authors participated in the design, conception, data gathering, writing, meaningful editing, suggestions, and feedback on the final manuscript. 
Funding Stryker Neurovascular; limited coordinating center administrative grant. No per patient cost was provided.

Competing interests IL is consultant for Metronic, Stryker, Penumbra, and Cordis. MM is consultant for Claret Medical, Nogueira-Stryker Neurovascular (Trevo-2 Trial Principal Investigator - modest; DAWN Trial Principal Investigator - no compensation, TREVO Registry Steering Committee - no compensation), Medtronic (SWIFT Trial Steering Committee - modest; SWIFT-Prime Trial Steering Committee - no compensation; STAR Trial Angiographic Core Lab - significant), Penumbra (3D Separator Trial Executive Committee - no compensation), Neurav (ARISE-2 Steering Committee - no compensation), Genentech (Physician Advisory Board - modest), Allm Inc (Physician Advisory Board - no compensation), EditorIn-Chief Interventional Neurology Journal (no compensation). SRS is a consultant for Stryker Neurovascular. OOZ is overall PI for TRACK - no compensation, Arise II - modest, Co-PI Therapy Trial - modest, Steering committee STRATIS registry modest.

Ethics approval Ethics approval was provided by Mercy Health St. Vincent Hospital institutional review board and the ethics committee at each participating institution.

Provenance and peer review Not commissioned; externally peer reviewed.

Data sharing statement There are no additional unpublished data from this particular study comparing the post marketing experience of the TREVO device with the industry sponsored prospective study. Additional questions are being addressed by other sub-papers currently under development.

Open Access This is an Open Access article distributed in accordance with the Creative Commons Attribution Non Commercial (CC BY-NC 4.0) license, which permits others to distribute, remix, adapt, build upon this work non-commercially, and license their derivative works on different terms, provided the original work is properly cited and the use is non-commercial. See: http://creativecommons.org/ licenses/by-nc/4.0/

(c) Article author(s) (or their employer(s) unless otherwise stated in the text of the article) 2018. All rights reserved. No commercial use is permitted unless otherwise expressly granted.

\section{REFERENCES}

1 English JD, Yavagal DR, Gupta R, et al. Mechanical thrombectomy-ready comprehensive stroke center requirements and endovascular stroke systems of care: recommendations from the Endovascular Stroke Standards Committee of the Society of Vascular and Interventional Neurology (SVIN). Interv Neurol 2016;4:138-50.

2 Powers WJ, Derdeyn CP, Biller J, et al. American Heart Association/American Stroke Association focused update of the 2013 guidelines for the early management of patients with acute ischemic stroke regarding endovascular treatment: a guideline for healthcare professionals from the American Heart Association/American Stroke Association. Stroke 2015;2015:3020-35.
3 Berkhemer OA, Fransen PS, Beumer D, et al. A randomized trial of intraarterial treatment for acute ischemic stroke. N Engl J Med 2015;372:11-20.

4 Goyal M, Demchuk AM, Menon BK, et al. Randomized assessment of rapid endovascular treatment of ischemic stroke. N Engl J Med 2015;372:1019-30.

5 Campbell BCV, Donnan GA, Lees KR, et al. Endovascular stent thrombectomy: the new standard of care for large vessel ischaemic stroke. Lancet Neurol 2015;14:846-54.

6 Saver JL, Goyal M, Bonafe A, et al. Stent-retriever thrombectomy after intravenous t-PA vs. t-PA alone in stroke. N Eng/ J Med 2015;372:2285-95.

7 Jovin TG, Chamorro A, Cobo E, et al. Thrombectomy within 8 hours after symptom onset in ischemic stroke. N Engl J Med 2015;372:2296-306.

8 Badhiwala JH, Nassiri F, Alhazzani W, et al. Endovascular thrombectomy for acute ischemic stroke: a meta-analysis. JAMA 2015;314:1832-43.

9 Mocco J, Zaidat 00, von Kummer R, et al. Aspiration thrombectomy after intravenous alteplase versus intravenous alteplase alone. Stroke 2016;47:2331-8.

10 Campbell BC, Hill MD, Rubiera M, et al. Safety and efficacy of solitaire stent thrombectomy: individual patient data meta-analysis of randomized trials. Stroke 2016;47:798-806.

11 Goyal M, Menon BK, van Zwam WH, et al. Endovascular thrombectomy after large-vessel ischaemic stroke: a meta-analysis of individual patient data from five randomised trials. Lancet 2016;387:1723-31.

12 Saver JL, Jahan R, Levy El, et al. Solitaire flow restoration device versus the Merci Retriever in patients with acute ischaemic stroke (SWIFT): a randomised, parallelgroup, non-inferiority trial. Lancet 2012;380:1241-9.

13 Nogueira RG, Lutsep HL, Gupta R, et al. Trevo versus Merci retrievers for thrombectomy revascularisation of large vessel occlusions in acute ischaemic stroke (TREVO 2): a randomised trial. Lancet 2012;380:1231-40.

14 Zaidat 00, Castonguay AC, Gupta R, et al. North American Solitaire Stent Retriever Acute Stroke Registry: post-marketing revascularization and clinical outcome results. J Neurointerv Surg 2014;6:584-8.

15 Nogueira RG, Zaidat 00, Castonguay AC, et al. Rescue thrombectomy in large vessel occlusion strokes leads to better outcomes than intravenous thrombolysis alone: a 'real world' applicability of the recent trials. Interv Neurol 2016;5:101-10.

16 Pereira VM, Gralla J, Davalos A, et al. Prospective, multicenter, single-arm study of mechanical thrombectomy using Solitaire flow restoration in acute ischemic stroke. Stroke 2013:44:2802-7.

17 Zaidat 00, Yoo AJ, Khatri P, et al. Recommendations on angiographic revascularization grading standards for acute ischemic stroke: a consensus statement. Stroke 2013;44:2650-63.

18 Nguyen TN, Malisch T, Castonguay AC, et al. Balloon guide catheter improves revascularization and clinical outcomes with the Solitaire device: analysis of the North American Solitaire Acute Stroke Registry. Stroke 2014;45:141-5.

19 Zaidat O, Liebeskind D, Jahan R, et al. Influence of balloon, conventional, or distal catheters on angiographic and technical outcomes in STRATIS. J Neurointerv Surg 2016;8:A3.2-A4.

20 Zaidat O, Castonguay A, Gupta R, et al. The first pass effect: a new measure for stroke thrombectomy devices. J Neurointerv Surg 2015;7:A2.2-A3. 